\title{
General Collective Intelligence and the Constraints to Group Decision-Making
}

\begin{abstract}
This paper addresses the question of how current group decision-making systems, including collective intelligence algorithms, might be constrained in ways that prevent them from achieving general problem solving ability. And as a result of those constraints, how some collective issues that pose existential risks such as poverty, the environmental degradation that has linked to climate change, or other sustainable development goals, might not be reliably solvable with current decision-making systems. This paper then addresses the question that assuming specific categories of such existential problems are not currently solvable with any existing group decision-systems, how can decisionsystems increase the general problem solving ability of groups so that such issues can reliably be solved? In particular, how might a General Collective Intelligence, defined here to be a system of group decision-making with general problem solving ability, facilitate this increase in group problem-solving ability? The paper then presents some boundary conditions that a framework for modeling general problem solving in groups suggests must be satisfied by any model of General Collective Intelligence. When generalized to apply to all group decision-making, any such constraints on group intelligence, and any such system of General Collective Intelligence capable of removing those constraints, are then applicable to any process that utilizes group problem solving, from design, to manufacturing or any other life-cycle processes of any product or service, or whether research in any field from the arts to the basic sciences. For this reason these questions are important to a wide variety of academic disciplines. And because many of the issues impacted represent existential risks to human civilization, these questions may also be important by to all by definition.
\end{abstract}

\section{Classes of Current Decision-Systems and Common Attributes}

Collective intelligence refers to a group's combined capacity and capability to perform a wide variety of tasks and solve diverse problems [3]. A collective intelligence algorithm is defined in this paper as a system of group decision-making that selects solutions which maximize individual or collective outcomes. Regarding organizational decision-systems, extensive studies have been conducted to identify and classify the different types [1] of such systems, and a great deal of research in the areas of collective intelligence, swarm intelligence, and other group decision-systems is currently ongoing [4],5],[6],[7],[8]. Additional studies have investigated whether groups, regardless of which decisionsystems they might use, can be observed to have an intelligence factor that is an emergent property of the group [9], and therefore that is distinguishable from the intelligence of individuals in the group. This proposed intelligence factor has been represented as a measure of the group's general problem solving ability.

But setting aside whether or not such a group intelligence factor exists in a particular group, the concept of general problem solving ability might benefit from clarification to understand how "general problem solving ability" might be defined in a way that is based solely on the function of problem solving [69], and therefore that is general in applying not only to individual human cognition (i.e. how individual humans solve human problems with human solutions), but also applying to other entities such as groups, or even other species. Any animal, for example an ant, might choose its own problem definition for a wide range of problems, and may respond to those problems with a wide range of solutions. An ant might see an orange as the problem of moving food. Or an ant might see an orange as the problem of it's nest being blocked. An ant might then see the solution as moving the orange. Or the ant might see the solution as moving the nest. Of course it's breadth of choice has a limit. There are categories of problems the ant can't define and categories of solutions the ant can't discover. In terms of these categories it has some breadth in problem solving. In terms of being able to chain smaller problems and their solutions together into solutions to larger problems the ant might also have some 
limit to the magnitude of it's problem solving ability. This combination of breadth and magnitude of problem solving ability somehow equates to a level of general problem solving ability. Similarly, when talking about human beings there are a wide variety of problems that humans can conceive of and solve, and there are categories of problems, and specific instances of problems that are outside the capacity of human-beings to conceive. For problems human beings can or cannot conceive, there also might be solutions outside the capacity of human-beings to discover. From this perspective, when we say an ant does not have general problem solving ability and a human does, this definition of general problem solving ability as a binary property that is either present or not present does not appear to refer to any intrinsic properties of problems or solutions, but instead appears to refer to some human perception related to problem solving, such as the presence or absence of human levels of general problem solving ability. If so, for general problem solving ability as a measurable factor to have an objectively defined meaning that is only dependent on the properties of problems or solutions and the ability to navigate between them (making it the same whether talking about individuals, groups, or other implementation details of the function of cognition), there must be a functional model of problem solving that makes the level of "general problem solving ability" objectively meaningful, and therefore potentially measurable in this implementation independent way. Such a model of problem solving is provided by a recently developed Functional Modeling Framework (FMF) [3]. In the FMF problems are defined as the gap between two points in a conceptual space. This conceptual space is defined at higher or lower resolution depending on the organism's capacity for abstraction, and is therefore also general rather than human-centric. The breadth of problem solving ability is defined as the level of ability to navigate from one concept to nearby concepts in all directions. The magnitude of problem solving ability is defined as the level of ability to navigate from one concept to far away concepts in a single direction, such as might be possible when chaining together multiple smaller solutions into a single larger one.

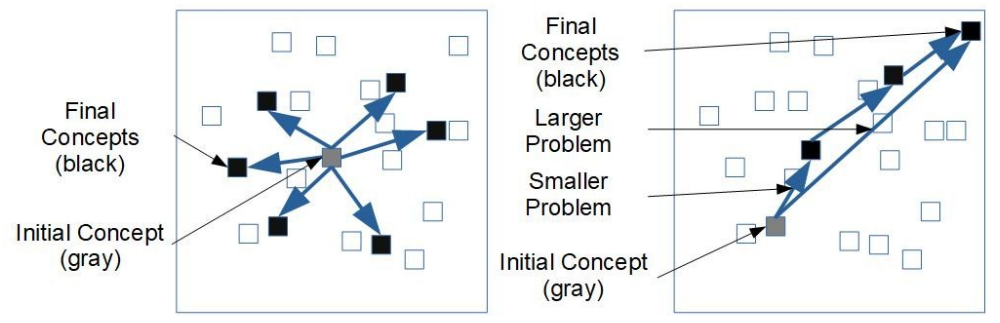

Figure 1: Problems are defined by gap between initial and final concepts. Breadth of problem solving ability is defined in the FMF as the capacity to navigate from an initial concept to many different final concepts. Magnitude of problem solving ability is defined in the FMF as the capacity to navigate from an initial concept to different final concepts through chains of reasoning executed sequentially. These chains of reasoning combine solutions to smaller problems into a solution to a larger problem.

In the FMF general problem solving ability is represented as the volume of paths through conceptual space that can be navigated per unit time. 


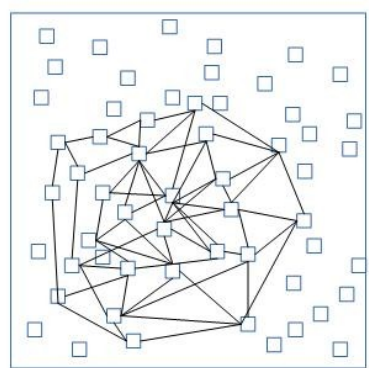

Larger volume of conceptual space navigated per unit time (higher general problem solving ability).

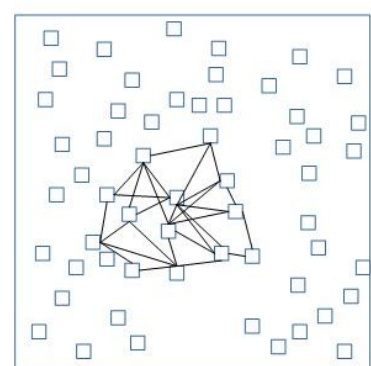

Smaller volume of conceptual space navigated per unit time (lower general problem solving ability).

Figure 2: General problem solving ability.

Currently, since human-like cognition has not been replicated, the only known intelligence with the capacity for human general problem solving is by definition individual human intelligence. Decisionmaking falls within general problem solving, which we will assume requires reasoning. All reasoning occurring in human beings has been categorized as falling into two categories, rational methodical reasoning (slow thinking), and intuitive reasoning (fast thinking) [10]. Intuitive reasoning is assumed here to be a black box in which solutions are based on estimation from past patterns through unknown mechanisms so that subjects might not be able to explain how such conclusions were obtained. But regardless of whether intuitive reasoning processes can be explained, functional models have been defined for both them and for methodical reasoning processes. If automation doesn't change the processes from one type to another, these functional models must still apply when those reasoning processes are automated in decision-systems.

In the Functional Modeling Framework, to gain general problem solving ability a system of cognition requires both a library of cognitive processes and the ability to choose which cognitive processes to execute in order to solve any problem. Assuming this functional model of cognition is complete, then if all decision-systems implement some subset of cognition, all decision-systems must then consist of some cognitive processes executed by a system with some or all of the functionality required for general problem solving ability. If the FMF can be confirmed to have the capacity to represent the entire human cognitive system with functional models, including all potential human cognitive processes, then being merely an automation of human cognitive processes, all decision-systems and all their decision-making processes can be represented with functional models as well. Because the FMF defines such functional models, because such functional models provide a simple definition of general problem solving ability, and because such functional models define the components required to attain that ability, it becomes possible to use such models to determine the categories of decision-making systems lacking those components, and therefore lacking general problem solving ability. The FMF defines some key terms necessary to this determination.

\section{Definition of Terms in the Functional Modeling Framework \\ Term \\ Definition}

Function

Process

Fitness
A function in the FMF is one element of functionality in the set of basic elements of functionality belonging to each adaptive domain.

A process in the FMF differs from a function in that it might receive multiple instances of input during it's execution.

Fitness to execute a single process or function in the FMF is the relative ability to execute a process or function of a functional system defined in the FMF. Functions are modeled as having inputs, executing within a context, and 
Fitness Space

Functional System

Well-Being

General Problem

Solving Ability

Functional Stability producing outputs associated with outcomes. Fitness is expressed in volume of outcomes per volume of inputs.

In the FMF each model of a functional system defines fitness as an $\mathrm{N}$

dimensional space in which each dimension is a property of fitness. In the change engine model of collective cognition described in this paper the dimensions of the fitness space of the collective cognition are defined by the properties projected fitness, targeted fitness, and actual fitness.

The functional systems defined in the FMF are the body, emotions, mind, and consciousness. The functional system in a first order collective are the collective body, the collective emotions that prioritize the motivations of the group, the collective mind, and the collective consciousness. The collective motivation directs focus to the activities most highly prioritized by the group. The collective mind (collective cognition) selects optimal outcomes according to the collective cognitive well-being. And the collective consciousness directs the collective awareness according to the collective conscious well-being. In the FMF well-being in each functional system is defined as the combined fitness of the functional system to execute all of its functions.

Representing a problem as the lack of a path between two points in conceptual space, representing a solution as such a path, and representing each path to have a common cross sectional area in conceptual space, general problem solving ability in the FMF is represented as the volume of conceptual space that can be navigated by the cognitive system per unit of time.

Stability as defined for functions in the FMF refers to stability of their fitness to achieve their outcomes. Stable functions do not spontaneously appear or disappear.

Table 1: Key definitions in the Functional Modeling Framework.

Modeling decision-processes as consisting of reasoning, the cognitive system is observed to have a number of properties that allow it to execute these processes. One such property is the ability to generalize all reasoning processes so that they are instances of a single class of solution to a single general problem. The ability to execute this single class would then enable the cognitive system to use an open set of reasoning processes (i.e. any available reasoning) to solve an open set of problems (i.e. any problem conceived). Assuming that the only general cognitive problem common to all humans is the problem of achieving well-being, then if the cognitive system requires well-being as a general problem to solve in order to gain general problem solving ability, then in order to have general problem solving ability the cognitive system must have a metric for the impact of both problems and solutions on that well-being. If the cognitive system is to continue to function in order to process any such reasoning then it also requires stability in its fitness to function. Because the thought process is not observed to spontaneously appear or disappear regardless of reasoning encountered or chosen, even though individual thoughts might come and go.

From the above arguments, though there are a great many collective intelligence algorithms for collective problem solving [4], as well as many other bio-inspired and other approaches to computing [8], then without testing each of them on every potential problem, existing decision-making systems can be observed to lack general problem solving ability by the following (non-exhaustive) criteria defining classes of problems they don't solve:

- Failure to Maximize Collective Well-Being: individual intelligence targets the problem of maximizing individual well-being [64]. If by analogy collective intelligence is 
distinguishable from the individual intelligence of members of the group in that it targets the problem of maximizing collective well-being, then in order to have the capacity to confirm that a system of collective well-being maximizes collective well-being within its capacity to do so, there must be a way of measuring that collective well-being. Approaches to CI that lack a methodology for incorporating measurement of collective well-being in determining fitness at performing tasks fail in this respect.

- Lack of Global Stability in Fitness: Of any given set of problem solving strategies, only a subset can be executed by a given system within the resources available to it. If a problem solving system is to reliably have the capacity to optimize outcomes, it must exhibit sustainable dynamics in its fitness to optimize outcomes. That is, it must select problem solving strategies it projects it can sustain to their conclusion, while also measuring results actually achieved, so investment can continue or shift to solving a more optimal problem, or to solving it with a more optimal solution, as required. Approaches to CI that lack a methodology for ensuring global stability of the fitness of the system to function in optimizing outcomes fail in this respect.

While there are other models of GCI [2], by the above two criteria, there may be no other existing models with this potential capacity for general problem solving ability.

Assuming it is possible to define algorithms in software or other solutions that orchestrate cooperation in groups so they have the capacity to reason coherently as a single collective entity, then if these two properties at minimum are required in order to have the capacity for reasoning and general problem solving ability, groups governed by these decision-systems must have these two same properties if they are to have the capacity for reasoning and general problem solving ability as well.

Because current group decision-systems lack a metric for even measuring collective well-being they cannot be said to maximize achievement of it. And because they lack a mechanism for measuring wellbeing (fitness), they cannot be said to have a mechanism for maintaining global stability in fitness. If both a metric for well-being, and global stability in the system of cognition, are required for reasoning, and if reasoning is required for general problem solving ability, none of these decision-systems can be said to have collective general problem solving ability as an emergent property of the group, rather than problem solving ability reflective of that of the individuals in the group. The caveat to this statement is that as explored earlier in this paper, the current definition of general problem solving ability (intelligence) can't necessarily be extended to other instances of cognition, including groups. However, as mentioned as well, functional modeling of cognition has enabled a more universal, objective model of general problem solving ability to be defined [11].

From this functional modeling perspective, lacking some of the cognitive functionality required to have general problem solving ability, or required to have general problem solving ability belonging to the group rather than any individual, such decision-systems therefore all fall into the class of Group Decision-Making Systems Addressing Specific Problems rather than General Collective Intelligence or GCI which are defined here as group decision-making systems with general problem solving ability that is an emergent property of the group.

As discussed, the presence of general problem solving ability implies the potential to solve any problem that is solvable within the capacity of the problem-solving system. The level of general problem solving ability implies some measurement of that capacity. Having the potential to solve a problem implies the absence of constraints that reliably prevent the system from doing so. One problem that might be solved is the general problem of how to define problems in a way that optimizes 
outcomes. Another problem that might be solved is the general problem of selecting optimal solutions for any problem that has been defined. Without general problem solving ability, we would expect a decision-system to be constrained against selecting entire classes of ways to define problems and to be constrained against selecting entire classes of solutions to those problems, even where optimal.

Defining “Group Decision-Making Systems Addressing Specific Problems” as a set of decision-making systems representing most or all current group decision-making systems, these systems were observed to lack these two attributes of having the ability to maximize collective well-being (fitness), and having the ability to achieve stability in fitness. The absence of these two attributes in turn was observed to be consistent with that absence constraining decision-systems against selecting entire classes of ways to define problems and consistent with that absence constraining them against selecting entire classes of solutions to those problems, even where optimal.

\section{Group Decision-Maximizes Global Stability in Constraints System Collective Fitness Through all Well-Being Functional States}

Collective No No

Intelligence (non

$\mathrm{GCI})$

Single AI (non No No

AGI) or Single

Expert Systems
Observations are consistent with CI solutions being constrained against choosing a different problem to solve [19]. In terms of the first attribute (wellbeing) a collective intelligence algorithm that for example selects an optimal mammogram diagnosis might increase its problem solving ability and improve healthcare outcomes far more if instead of answering "does this image contain cancer?" it could answer "what should the patient do to maximize their well-being?" In terms of the second attribute (stability) a collective intelligence algorithm might increase its problem solving abilities if no matter what problem it solved (i.e. no matter where it navigated in conceptual space), it could continue to solve some problem definable from that new location in conceptual space, where that new problem created enough value for continued problem solving to be sustainable. By introducing this ability to continually execute its capabilities, and to continue to increase the magnitude of impact on any given problem as it did, the value of the system could increase to the point at which it was self-sustaining and therefore stable.

Observations are consistent with AI solutions being constrained against choosing a different problem to solve by the inability to generalize [20]. Expert systems are constrained against choosing a different problem to solve by the difficulty of encoding all possible problems. In terms of the first attribute (well-being) a solution that selects an optimal diagnosis of a specific condition might increase its problem solving ability and improve healthcare 


Decentralized No No
(Consensus
Voting)
Decision-Making

Centralized No No (Autocratic)

Decision-Making outcomes far more if instead of answering "does this image support the diagnosis of the specified condition?" it could maximize their well-being as above. In terms of the second attribute (stability) autocratic decision-making might also increase it's problem solving ability in same way as CI.

Observations are consistent with consensus voting being constrained against selecting an optimal solution (decision or decision-maker) that lacks mind share. In the case of selecting a decisionmaker this means it is constrained against candidates unable to campaign effectively [21]. In terms of the first attribute (well-being) consensus voting might increase its problem solving ability by objectively defining the impact of a choice on wellbeing. In terms of the second attribute (stability) consensus voting might also increase its problem solving ability in same way as CI. But such voting systems do not yet exist.

Observations are consistent with autocratic decision-making being constrained against selecting solutions that do not align with the cognitive biases, beliefs, personal interests, lack of knowledge, time availability, or other limitations of the decisionmaker. In terms of the first attribute autocratic decision-making might increase its problem solving ability by objectively identifying the decisionmaker with the greatest aptitude and inclination to select choices that maximize impact on collective well-being. In terms of the second attribute autocratic decision-making might also increase it's problem solving ability in same way as $\mathrm{CI}$. But such decision-maker selection systems do not yet exist. In most cases autocratic leaders may select themselves [22].

Table 2: Sets representing most or all current group decision-making systems. These systems were observed to lack at minimum the two specified attributes required for human general problem solving ability and were observed to be constrained against selecting entire classes of ways to define problems and tend to be constrained against selecting entire classes of solutions to those problems, even where optimal.

\section{Constraints to Current Decision-Systems}

If in the absence of the above two attributes decision-systems are reliably constrained (greater than $50 \%$ probability of being constrained) against selecting entire classes of problems or solutions, and if general problem solving ability requires the absence of such constraints, then this absence suggests that problems exceeding these constraints are by definition not reliably solvable (less than 50\% probability of being solved) by a decision-system. Given that one constraint on group problem-solving is that problems of well-being are not reliably solvable by a decision-system that lacks a way of measuring well-being, this suggests that any categories of collective problems that involve collective well-being 
might not be reliably solvable with current decision-systems lacking a way of measuring collective well-being. This includes collective problems such as poverty, the environmental degradation often linked to climate change, and other existential threats to human civilization.

In addition to the properties of decision-systems that constrain them so they lack general problem solving ability, some of the properties of problem definitions and solutions that constrain group decision-making are:

\section{Properties of Problem Definitions and Solutions that Constrain Group Decision-Making}

The perceived individual interests of decision-makers don't align with the selection. Because these interests don't necessarily align with group interests, this negatively impacts the group's ability to solve the general problem of maximizing the group's interests [70].

Cooperation involves too many interactions (such as a Nth order barter transaction) for the chains of cooperation to be discoverable without automation.

Problems or solutions too complex to be understood by group members [71], [72].

Problems or solutions too multi-disciplinary [73].

Solutions require too much work to be feasible for the group, such as involving the evaluation of too many options [74].

Problems or solutions conflict with cognitive biases of the group, such as belief systems [75].

Require a large amount of resources and therefore require the buy-in of one or more entities that are large enough to have such resources, but at the same time the solutions require a flexible approach that conflicts with the rigid policies large organizations tend to have.

Table 3: In addition to decision-systems being constrained by their lack of the properties required for general problem solving ability, they are also constrained from defining specific problems or from discovering specific solutions by the above properties of problems and of solutions.

\section{Classes of Collective Problems that are Not Reliably Definable or Solvable by a Decision-System} The Functional Modeling Framework represents individual and collective systems of cognition as having general problem solving ability, and suggests the functionality required to achieve that general problem solving ability. The key functional elements of the FMF that together are proposed to help ensure convergence on achieving all capacities related to general problem solving fitness, including definition of the optimal problems, selection of the optimal solutions, and use of both in their optimal domains, are below:

\section{Component of GCI Model Description}

Functional modeling

(problems and solutions)

Functional decomposition

Functional domain bridging Different domains in which different functions are more fit in achieving

Functional fitness

Functional stability

Functional adaptation the same purpose are identified. These domains are bridged by using a set of weights which identify the best function in each domain.

Components are modeled only by function to remove prejudice for or against any given implementation.

Functional components are decomposed into their most basic functional building blocks for reuse.

Every functional component is assigned some projected and actual fitness in achieving its function.

For functional components to persist they must display some degree of stability in fitness to function.

For functional components to persist in a changing environment they must have the ability to adapt their function. One of the most important domains of adaptation is cooperation. Functional components must 
interact according to the principles of decentralized cooperation in order to maximize outcomes for each. Functions must be prioritized according to the principles of centralized cooperation in order to maximize outcomes globally.

\section{Table 1: Components of the General Collective Intelligence model.}

In addition to these functional elements of the FMF, there are also infrastructure elements such as the frameworks for representing the conceptual space, relative fitness, and other aspects of cognitive functionality. Assume decision-systems have some subset of the functionality of cognitive systems with general problem solving ability. Consider functional models of those decision systems as well as considering functional models of the problems and solutions they solve. For each element of functionality of cognitive systems, classes of problems can be defined that are not be solvable without that element of functionality, and by extension are not solvable without a decision-system having that element of functionality. For each of these categories the table below provides data consistent with the observation that these problems have not been solved the majority of the time and therefore are not reliably solvable in practice.

Defining the problem or solution in terms other than functional ones.

\section{Class of Problem}

Specific Examples

Failure to define a common

way of representing and comparing renewable energy solutions based on their function, and the resulting failure to escape the dogmatic insistence on one category of solution or rejection of another [17].

Defining the parts of problem Failure to define a common or solution in a way that is specific to a given implementation of a solution rather than general to all solutions.

Defining the problem or solution in a way that does not make use of all previous ones way of representing and comparing models of consciousness or cognition based on their function, and the resulting failure to converge on a common understanding of the problems being solved or to converge on a common understanding of the solution [12].

Ineffectiveness of employment programs that don't learn from the lesson of other employment programs that training as a means of employment creation does no create employment unless training for an available or anticipated opening [18].
Properties Common to Failed Problem Definitions

Lack of universal methodology for functional modeling of problems and/or solutions.

Lack of universal methodology for functional decomposition (decomposing functionality into basic components).
Lack of universal methodology for functional domain bridging (identifying domains where a given entity functions best as well as how to transition between those domains, and how to store this ot information in a way that doesn't violate the principles of decentralization) 
Defining the problem or solution according to any criteria other than what works

Defining the problem or solution specifically rather than as lack of stability in continuing to address all problems or solutions
The ineffectiveness of

Lack of universal methodology for development aid programs on functional fitness (optimizing function). growth and their continued design according to policies determined by factors other than effectiveness [15].

The ineffectiveness of global Lack of universal methodology for cooperation to impact the functional stability (ability to sustain environmental degradation function).

associated with climate change and the inability to evolve cooperation that converges on collective wellbeing in a more stable way [13], [14].

Defining the problem or solution as static rather than changing
Setting educational policies asLack of universal methodology for a one time exercise rather functional adaptation (ability to converge than establishing a process foron optimal result despite changing problem continual updating results in definition and/or changing solutions). outdated policies, and as a result, ineffective spending per unit of educational outcomes [16].

Table 4: Categories of collective problems with data showing that these problems are not reliably solvable in practice.

\section{Adaptive Decision-Making Systems}

Intelligence has been defined in psychology as the general problem solving ability that all conscious human beings have some degree of, with the possible exception of those with some form of cognitive impairment. The intelligence quotient or IQ has been defined as a measure of the level of this ability [2]. The recently developed Functional Modeling Framework (FMF) [3] for modeling cognition and other functional components of the human system represents human beings as consisting of a hierarchy of adaptive processes with which humans adapt to solve problems posed by the environment. Each of these processes is defined as operating within a different adaptive domain and as being implemented by a functional system, or being implemented by a functional component that might be present in multiple functional systems. These domains are defined as being composed of a set of basic functions. All the states accessible to each functional system through it's functions then form a functional state space that the system can be modeled as moving through. In the case of cognition, the cognitive system is represented as moving through a space of concepts (conceptual space) that forms the functional state space of the cognitive system.

The adaptive properties of individual human intelligence that are required to have some degree of general problem solving ability, are some ability to choose the optimal solution for cognitive problems, and some ability to decide which cognitive problems to solve. A number of classes of individual problems can be determined to be reliably solvable by some individual because they are solved by the majority of individuals, though they might not be reliably solvable by any given individual.

Individual Problems

Access to Employment
Percentage of Population Solved by

The percentage of people in the US who were eligible for 
Access to Healthcare

Food Security

Access to Housing employment and who were employed in 2018 was 96.4 percent [18]

The percentage of people in the US with health insurance coverage for all or part of 2018 was 91.5 percent [19] In $2018,88.9$ percent of U.S. households were food secure throughout the year.

The overall homeless population on a single night represents 0.2 percent of the U.S. population. Therefore $99.8 \%$ of people in the US on a single night have solved the problem of homelessness.

Table 5: Individual problems observed to reliably be solved by some individual.

These problems are then reliably solvable (greater than 50\% probability of being solved) by individual human intelligence as a class of decision-making system with the range of general problem solving ability observed in humans. A decision-making system that is like human intelligence in having the all the functions needed to gain the ability to adapt to choose optimal problem definitions therefore has the ability to define these problems in a way that is optimal enough for the problems to be reliably solvable. And decision-making system that is like human intelligence in having all the functions required to gain the ability to adapt to choose optimal solutions therefore has the ability to solve those problems in a way that is optimal enough for those problems to be reliably solable.

If the set of functionality defined in this functional model as being required for general problem solving ability is complete, then any decision-making system having all of this required functionality must also make the above classes of problems reliably solvable (greater than $50 \%$ probability of being solved). If the functional model of cognition is implementation independent as believed, and therefore also applies to groups, then if a collective decision-making system has this functionality, these classes of problems that can be reliably solved by individual humans must also be reliably solvable for the collective as a single entity.

If all the attributes required to have this range of human general problem solving ability can be defined, then since observations are consistent with this range of general problem solving ability being able to make the above problems reliably solvable, then regardless of whether or not any specific attributes can be identified as being required to solve any specific problem, all cognitive systems implementing all those attributes must have the ability to reliably solve these problems.

However part of gaining general problem solving ability is solving the problem of ensuring pervasive usage. Because any decision-making system, no matter how good, can't improve problem solving ability if it isn't used. Ensuring usage isn't as simple as defining rules. Even if usage of collective intelligence is mandated, humans don't always follow such rules. Rather than defining a system of enforcement based on rules that can be broken, a more robust approach might be to define a system in which choice of computational collective intelligence solution, choice of method of enforcement, or choice of anything else, naturally converges on whatever works best. The Functional Modeling Framework referred to in this paper proposes to identify a set of elements with the capacity to reliably achieve this convergence, and the model of General Collective Intelligence (GCI) based on the FMF that is described in this paper proposes to implement those elements. This model of GCI has been called the "change engine" because it is proposed to have the potential capacity to target any collective problem, the potential capacity to significantly increase the probability of achieving impact on that problem, as well as the potential capacity to significantly increase the magnitude of impact achieved, and the potential capacity to use the value of these increases in impact to make impact financially self- 
sustaining once launched at any scale of impact that can be absorbed by the group, so that it becomes an engine for massively transformative change [59].

\section{The Change Engine Model of General Collective Intelligence}

The criteria for general problem solving ability identified in this paper suggests that a system of General Collective Intelligence or GCI must have the capacity to define any problem it has the capacity to conceive, and to solve it with any solution within it's capacity to concieve. The properties

determining the capacity of a cognitive system to conceive are explored elsewhere, where the cognitive system is as defined within this framework. Assuming that every collective decision-making system has functionality within the capacity of a GCI to represent, this in turn suggests that a GCI must have the capacity to leverage any decision-making system, and to leverage any problem definition used by such a decision-making system, in gaining that general problem solving ability. For this to be the case, such problem definitions and such decision-making solutions must be added to a library so they can be available for a GCI to use. And a GCI must be able to navigate that library in a way that provides the functionality required for collective cognition as defined in the FMF.

A theoretical model of collective cognition potentially satisfying these conditions is presented elsewhere. For each element of functionality required by cognition as defined in the Functional Modeling Framework, the model defines a boundary condition that any potential solution must satisfy. The model is a solution that potentially satisfies all of these boundary conditions, along with satisfying criteria for that solution to be applicable to a system of collective cognition (a General Collective Intelligence or GCI).

\section{Synthesis of the Concepts Surrounding General Collective Intelligence}

Collective intelligence refers to a group's combined capacity and capability to perform a wide variety of tasks and solve diverse problems. A collective intelligence algorithm is defined in this paper as a system of group decision-making that selects solutions which maximize individual or collective outcomes.

The FMF [3] aims to create the capacity to represent and compare models of individual and collective cognition. The model of general collective intelligence discussed in this paper has been defined within the FMF and therefore is proposed to have the potential for collective cognition. Since collective cognition is defined as having general problem solving ability, this model is proposed to be unique in having the capacity to reliably define and solve problems through the ability to converge on selecting solutions that maximize collective outcomes in problem domains where other models are proposed to face potentially hidden constraints that might prevent them from having the capacity to converge on optimal solutions, or any solutions at all. This model is also proposed to be unique in having the capacity to be applied in all domains within human conception, that is, in having the capacity for collective general problem solving ability that can be applied to any conceptual domain ("general collective intelligence").

From this perspective the FMF defines conventional Collective Intelligence (CI) as a group process that is analogous to narrow AI in that it solves a specific problem. The FMF defines collective cognition (General Collective Intelligence or GCI) as being analogous to Artificial General Intelligence (AGI) in that it has general problem solving ability. In a GCI this problem solving ability is proposed to be collective in that it is intrinsically aligned with the well-being of the group, by analogy with the intelligence of individual members of the group, which is intrinsically aligned with their individual interests [31], [32]. The difference might be commonly understood as avoiding hypocrisy. Where individual self-interest may lead to decision-makers to excuse themselves from decisions that they 
enforce on the rest of the group, collective interests are defined as those aligned with decisions that maximize collective well-being without heed to any individual interests [33].

Part of this approach to collective intelligence is creating common functional models of problems, and creating common functional models of the collective reasoning processes that might serve as solutions [34]. Such collective reasoning processes include the collective intelligence algorithms that might find opportunities for members of the group to achieve individual benefit by cooperating to implement reasoning that benefits the entire group. With such algorithms to facilitate automation where possible, cooperation in a collective intelligence might be computer-driven, potentially enabling the collective search for optimal solutions to occur at massively increased scope and scale.

In defining functional models of solutions in the conceptual space that forms the functional state space of the system of collective cognition within the FMF, a solution is represented as a path through the functional state space.

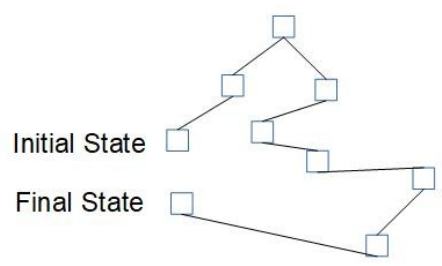

Figure 3: A collective reasoning process is a path

through the collective

conceptual space.

This path is a collective reasoning process that operates within a given context defined in terms of concepts, and that acts on a set of input concepts representing the initial state of the cognitive system in the conceptual space, to produce a set of output concepts representing a final state in that space. This collective reasoning is composed of a sequence of reasoning processes, each with their own inputs, outputs, and context, and each of which is represented by a segment of that path.

The overall output of the reasoning is related to some outcome so reasoning might potentially be optimized to increase those outcomes [35]. If executing each segment of a path representing a solution has a cost in terms of decreased fitness of the cognitive system, then solution processes might be redesigned algorithmically to follow a more optimal path through this space that minimizes cost of execution while maximizing value of the outcome achieved in terms of that same cognitive fitness.

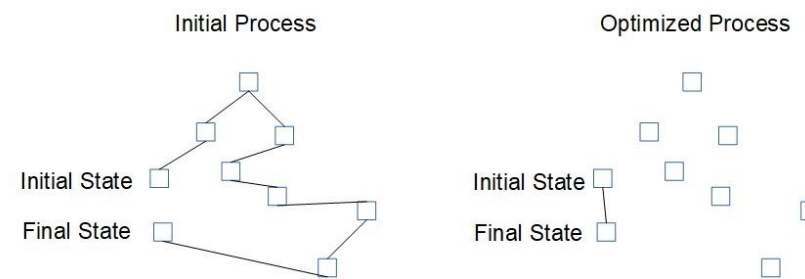

Figure 4: Optimizing collective reasoning processes by choosing a more efficient path through the collective conceptual space. 
The above diagram provides some insight on how collective intelligence algorithms might potentially increase problem solving ability in this way [36]. Taking the path from initial state to final state alone into account, if the volume of conceptual space that can be navigated per unit time to achieve greater cognitive fitness is related to the general problem solving ability of the collective, then by following more optimal paths general problem solving ability might potentially be increased through this optimization [37],[38].

General problem solving ability of the collective might also be increased by increasing the rate of outcomes.

Figure 5: A collective reasoning process that is a solution to a collective problem represented as a series of reasoning processes.

Representing a collective reasoning solution as implementing a series of such paths (collective reasoning functions) as above, we can increase the rate of outcomes by executing functions in parallel [39], [40]. We can execute functions in parallel when they are defined so that execution of one instance can be decoupled from execution of another.

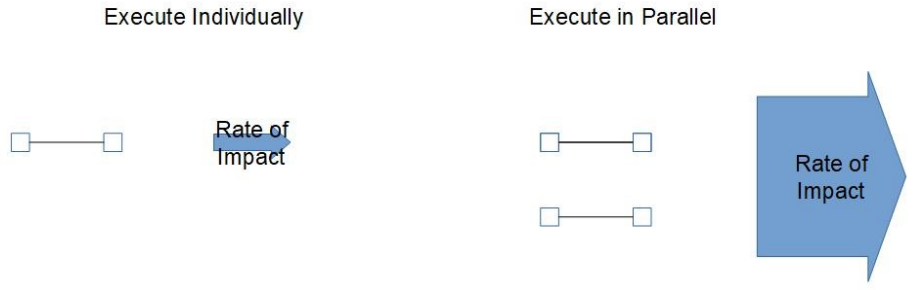

Figure 6: Collective reasoning processes scaled through parallel execution.

The volume of reasoning outcomes that might be achieved per unit of input and per unit of time is one measure of effectiveness in problem-solving, which is in turn related to intelligence [41], [42], [43]. We can potentially increase the magnitude of reasoning outcomes or transform reasoning outcomes to different ones by executing collective reasoning functions in series [44]. We can execute functions in series when one function acts on the outputs of another, so that execution of functions can be coupled together. In the case that the functions also are additive (i.e. $f(x+y)=f(x)+f(y)$ such as is the case with consecutive electrical transformers that increase voltage), then consecutive execution in series might increase the magnitude of outcomes.

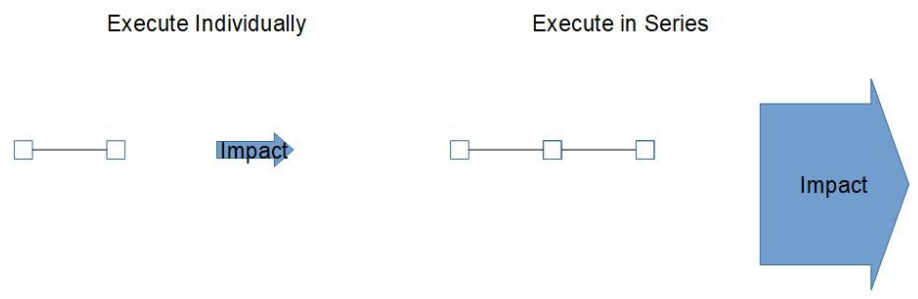

Figure 7: Collective reasoning processes scaled through serial execution. 
Increasing the volume of outcomes achieved per unit of input and per unit of time might also be achieved through increasing the resources available to execute collective reasoning processes. Some relevant resources are the collective reasoning processes themselves, the information that must be input into them, and the cost of accessing this information or creating or executing these processes. All resources might be increased. For example, by finding all other instances where the a reasoning process is used by individuals, and creating value through cooperating to save costs by generalizing such processes so they might be reused in collective reasoning, the collective reasoning available to a system of collective cognition, and therefore its effectiveness and in turn its general problem solving ability, might be increased.

Create and Use Individually

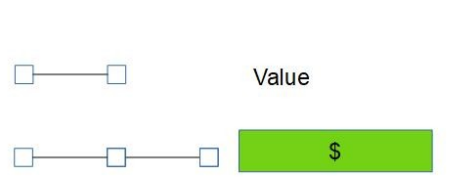

Create and Use Cooperatively

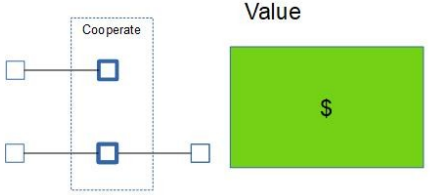

Figure 8: Outcomes scaled through cooperation.

Typically, reasoning processes that might be used by the group might be distributed over the individuals in group. Collective problem solving is a jigsaw puzzle. Each individual in the collective is a piece of the puzzle that may have some reasoning functionality others don't have. Finding the right reasoning processes is in part the "Algorithm Selection Problem" [45],[46],[47],[48].

Distribution of Functions

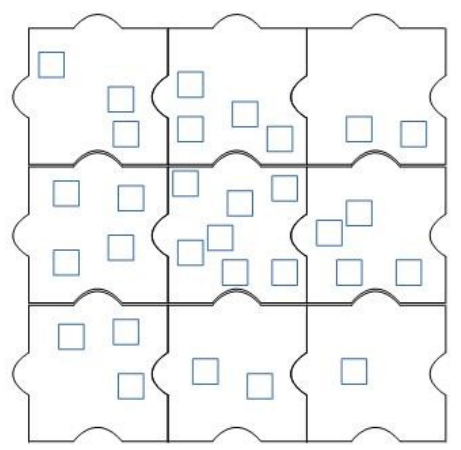

Figure 9: Reasoning processes represented as functions distributed among squares representing each group member.

Reasoning processes also require information. Each individual may have some information others don't have [49],[50],[51]. 


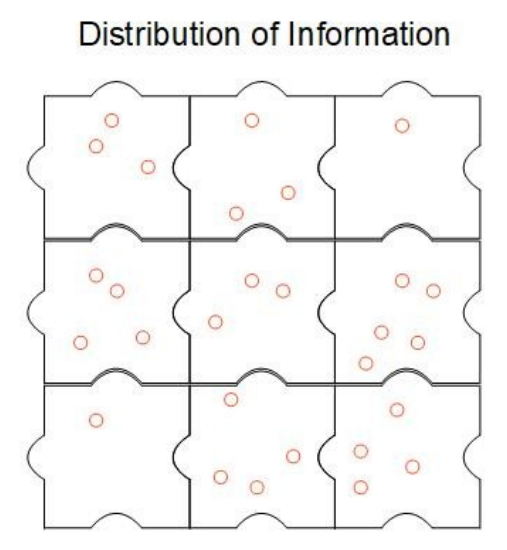

Figure 10: Required input to reasoning processes represented as information distributed among squares representing each group member.

Through the cooperation in a collective intelligence algorithm it may be possible to increase collective problem solving ability by enabling each individual in the collective to provide the piece of the jigsaw puzzle (the reasoning functionality or information) that others are missing.

But providing access to more reasoning processes or information alone might not be sufficient to significantly increase problem solving ability. There are limits to the volume of information that any individual in a group can access.

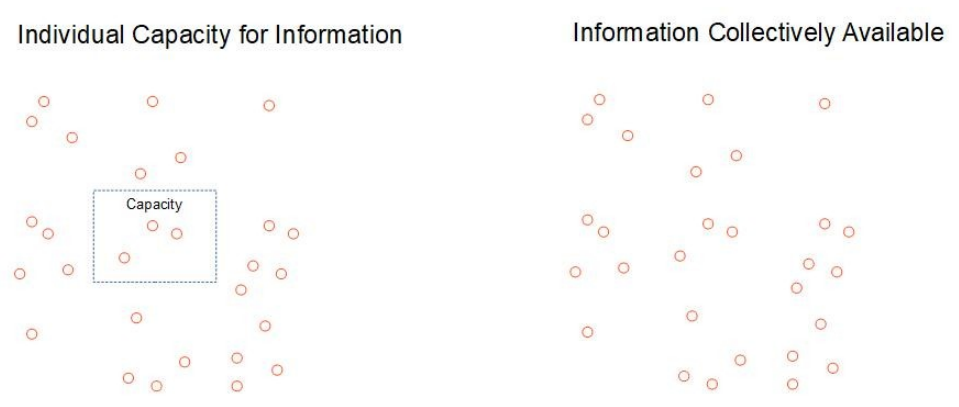

Figure 11: The capacity of individuals in the group for information might be far less than the information available in the group.

And there are limits to the volume of collective reasoning processes that an individual can navigate. The number of collective intelligence, bio-inspired computing [4], [52], [53],[54],[55] and other computational solutions for group decision-making in existence today may already be unmanageably large in comparison to the ability of any one individual to understand and choose between all of them. 
Figure 12: The capacity of individuals in the group to navigate reasoning processes might be far less than the reasoning available in the group.

Furthermore, these capacities might not align. In other words, the information required to navigate a given reasoning process might not be present with the reasoning process itself.

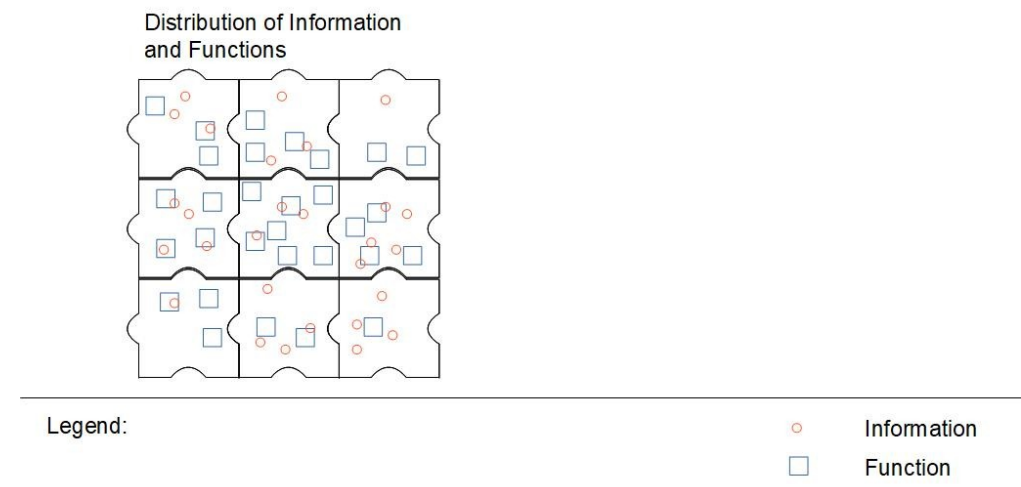

Figure 13: Combined distribution of reasoning processes and information among individuals in the group.

As a result the limitations in the capacity of individual group members to process information and to navigate reasoning might be far lower than the information and reasoning processes collectively available.

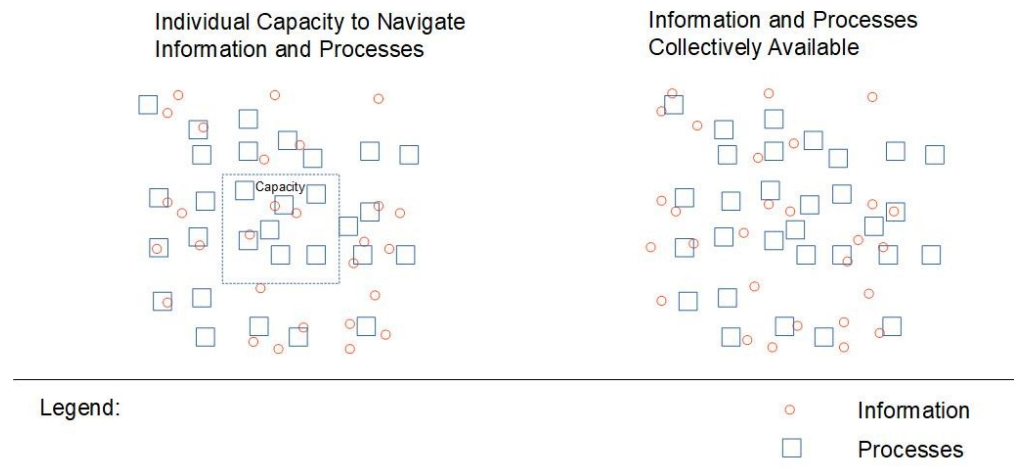

Figure 14: The limits created by the capacity of group members might be far smaller than the information and reasoning available.

Rather than attempting to navigate all information and reasoning processes available to the collective through having individuals in the group manually perform the necessary comparisons to find optimal reasoning processes as well as the optimal information to use in them, a more scalable approach might 
be to find a way to automate such comparisons through a universal and objective mechanism for defining problems and for measuring fitness in solving them. Being objective such a mechanism might be automated so that it is more reliably possible to compare the fitness of all available approaches in solving any problem.

Such a universal mechanism for comparing fitness in problem-solving might be critical in removing biases in the collective cognition or other barriers that might tend to restrict groups from making optimal decisions [56], [57], as is proposed here. One example is any case in which group decisionmaking might rely on consensus or other mechanisms that could potentially restrict groups from navigating all the domains of expertise of the group, rather than being limited to the domains of expertise common to all decision-makers. In other words, if every individual in the group understands hammer functionality, consensus can be used to identify problems as the need for a nail. But if only one individual in the group understands screwdriver functionality, consensus cannot necessarily be used to identify problems as the need for a screw.

Domains of expertise

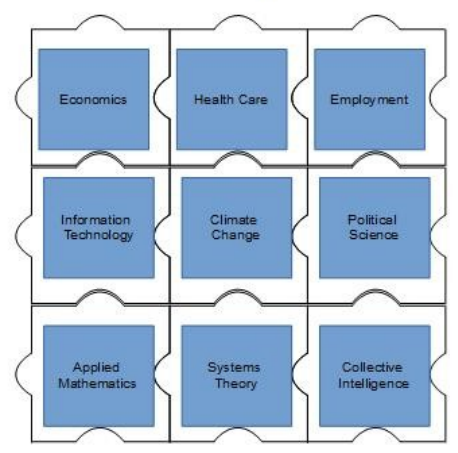

Figure 15: A general

collective intelligence must

be able to navigate all the

domains of expertise of any

individuals in the group.

Another critical part of collective intelligence is creating incentives to participate in collective reasoning processes. If fitness of the collective cognition has any dependence on access to cognitive resources, this participation could potentially enable a collective intelligence algorithm to gain fitness by harnessing the resources of the entire group, rather than being limited to the resources controlled by whatever subset of the group acts as decision-makers. 


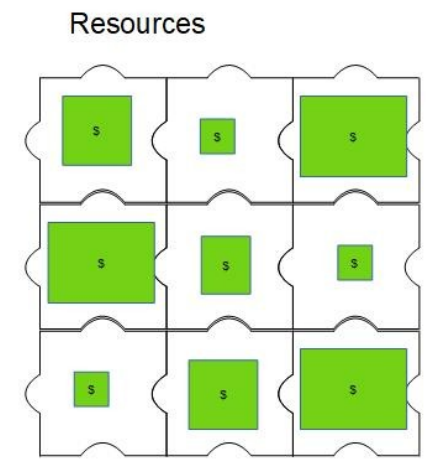

Figure 16: A general

collective intelligence must

have the capacity to harness

all the resources of the

group.

Through this increase in access to resources and information domains, collective intelligence might potentially increase the group's ability to achieve impact on general collective problems, where again that impact is defined in terms of volume of reasoning outcomes per unit time per volume of inputs.

Of course regardless of whether there are collective intelligence based group decision-making systems that might remove the limits to the volume of information and processes groups can navigate, even the best system decision-system can't be effective if it simply isn't used. So part of achieving optimal outcomes is also finding a way to ensure it is. Anecdotally, collective intelligence algorithms in general are not used pervasively in all group problem solving today [58], so despite the potentially large number of collective intelligence algorithms in existence, none has yet achieved pervasive use. If a system of collective decision-making cannot work if it is not predominantly used, then ensuring use of collective intelligence must be part of gaining general problem solving ability with collective intelligence. Without having to do an exhaustive literature search of all collective intelligence algorithms, if no collective intelligence algorithms are being used pervasively, then by definition none have implemented all of the elements of collective intelligence required to solve the problem of ensuring pervasive usage. If solving the problem of pervasive usage is part of general problem solving ability, then by definition no existing models of collective intelligence have implemented that ability.

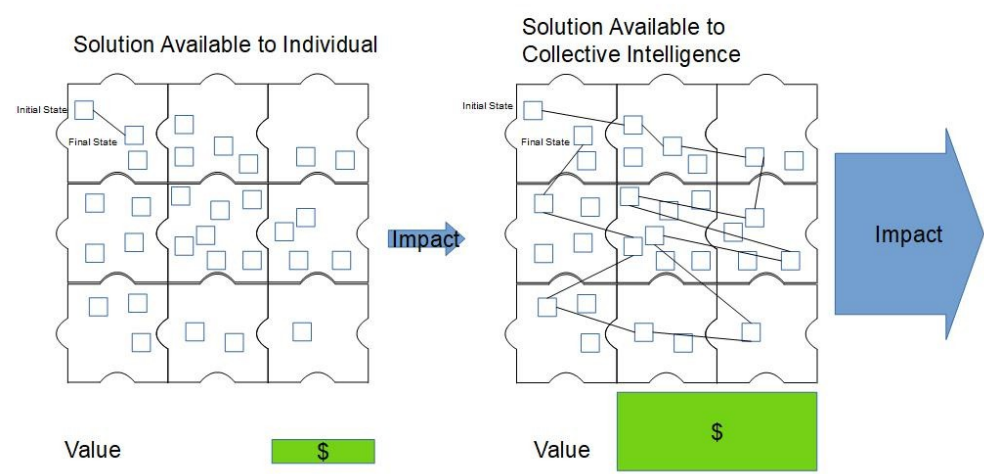

Figure 17: In order to be said to increase group intelligence, a general collective intelligence must increase the impact that can be achieved with the cognitive resources of the group. 


\section{The Implications of General Collective Intelligence on all Group Decision-Making}

Without decentralization in all of the layers provided by the adaptive processes and other components in the FMF, the forces driving centralization are proposed to effectively centralize any processes, including those automated by software platforms. Because ongoing work in game theory modeling suggests that the interests of a centralized decision-maker essentially impose a "tax" on all interactions, even those not involving that decision-maker directly, resources and decision-making power are proposed to gradually accumulate so decisions become aligned with their interests. In this way, these forces are proposed to drive operation, design, servicing, and all other processes along the entire product or service life-cycle, to become aligned with the interests of a subset of decision-makers rather than with optimizing collective outcomes. In the case of bitcoin, for example, while the blockchain provides decentralization in some of it's processes of operation, the centralization of value enables one user to speculate away the value contained in another user's bitcoin. If devaluation of paper currency by government policies was any motivation to use such digital currencies, being vulnerable to speculation that has the potential for even swifter devaluation is far from an optimal outcome for the group as a whole. Leveraging the blockchain to do a better job of designing a decentralized store of value is certainly achievable. But the process of designing bitcoin was a centralized one, and by the reasoning that centralized processes tend to become aligned with the interests of their owners, this likely served the interests of the designer very well by making him quite rich.

Without a mechanism for reliably decentralizing all processes (including design), the work mentioned above that is currently under way involving game theory modeling appears to suggest that increasing alignment with centralized interests is the natural balance of any software platform, even if it's main aim is decentralization. In other words, if all the required layers of decentralization are not present, decentralization solutions may simply move centralization elsewhere. Whether all these layers of decentralization are be required in every process of the entire business life-cycle of all products and services used by the GCI if the collective is to have the capacity to maximize collective outcomes, and whether GCI is required to achieve this pervasive decentralization, are issues for further study.

If so, this pervasive importance give GCI powerful implications that cross industries. Because unlike in natural systems, the technology of human groups might free the generation of inequality from being limited by what an individual organism can consume or store. If so, any such unbounded inequality and the concentration of resources resulting from it could potentially drive an unbalance that centralizes decision-making power to the point at which the decentralization that may be required to implement a decentralized system of decision-making like a GCI, and therefore the decentralization required to have the capacity to reliably optimize or even achieve collective outcomes, might no be longer achievable. This potential technology fueled tendency towards centralization has been called the "technology gravity well". Because of it, all the layers of decentralization provided by this GCI model are proposed to be critical to achieving any stable decentralization at all.

Consensus-based voting is another example. GCI is proposed here to be required for groups to reliably converge on a single line of reasoning in a decentralized way, and for the collective reasoning necessary for decentralized decision-making to be reliably achievable. Consensus-based voting may appear to be decentralized decision-making, but without GCI to enable collective reasoning, group opinions might be forced to rely on other mechanisms such as collective emotion in order to converge, particularly as groups get larger. Because collective emotions are most effectively directed by individuals with a gift for using oration to move crowds, this could effectively centralize consensusbased voting in key areas so that it is aligned with the interests of the decision-makers moving the crowd, rather than being aligned with the crowd itself. 


\section{Facilitating Ubiquitous Access to Data and Processes as a Boundary Condition for the Cooperation Domain in Group Decision-Making}

In the domain of adaptation through cooperation within the FMF, the principles of intelligent cooperation ensure a balance between processes driven by decentralized cooperation and processes driven in a centralized way. Group cooperation must be orchestrated in a decentralized way when required to maximize outcomes for all participants. And group cooperation must be orchestrated by a centralized actor or subset of actors when required to prioritize the functions of a subset of participants in order to maximize total outcomes. Because all processes may have some interaction with other processes, all processes may be connected within only a few degrees of separation. For this reason the forces of centralization potentially only need to be present in a few processes in order for outcomes in all processes to be dominated by centralization. By contrast, all of the many levels of decentralization must be present in order to balance these forces of centralization so that maximizing collective outcomes is reliably achievable.

As outlined in this paper, all entities, whether individuals or groups, must have the capacity to adapt through all the adaptive domains defined by the FMF in order to have general problem solving ability. And entities must have at least human-like general problem solving ability in order for them to have the capacity to reliably address problems that human-like levels of general problem solving ability can be demonstrated to reliably solve.

The boundary conditions on cooperation that all processes must be constrained by in order for those processes to have the capacity to adapt within the cooperation domain of the FMF are the same principles of intelligent cooperation described in this paper. In processes for accessing data these boundary conditions require providing all members of the group with decentralized access to all data and all processes by which decisions can be made. By the reasoning presented earlier in this paper, without this decentralized access all design and other processes involved in the entire life-cycle of any product or service would in this aspect be centralized. And a centralized repository of data and processes might naturally tend to become aligned with the interests of its owner rather than with optimal collective outcomes. Therefore, rather than accessing collective knowledge through such a centralized repository, the principles of collectively intelligent cooperation in the FMF within which the current model of GCI is defined, instead suggest cooperation through a mechanism of massive decentralized queries in which each individual has their own private repository of data, identities, applications, and other automated processes.

Providing ubiquitous access to data and processes also requires a common semantic representation so that data definitions can't be centralized. The current model of GCI defines a model for semantic representation of data and processes (the eXtensible Domain Modeling Framework or XDMF which is currently under development). Again, the XDMF is defined using the same human-centric modeling approach to represent the way human beings intuitively describe these entities, in the attempt to create representations that can be intuitively understood in natural language. This semantic modeling of each user's data, identities, and applications acts to "open" them so they are available for use in collective processes.

Leveraging this semantic modeling along with the principles of collectively intelligent cooperation opens participation in collaborative processes to all prospective parties. Functional decomposition breaks those processes into objectively defined components that any participant capable of filling the required role can potentially cooperate to execute. And semantic modeling enables outcomes of interactions within those collaborative processes to be stored in a common way. Furthermore, this functional decomposition and semantic modeling decouple processes, enabling parts of processes to 
run in parallel, so a GCI can orchestrate execution of those functions collaboratively at far greater speed and scale. By creating a private intelligent agent for each user that provides access to the user's data on the user's behalf according to policies they specify, where interactions follow processes that can be modeled, then if an individual's responses follow policies that can be implemented by a trusted intelligent agent that responds far more quickly on the individual's behalf, the number of those agents can be multiplied, and the rate of interactions can be further scaled.

Most or all of the required components for such agents may already exist. Defining requirements for each component, and defining a metric for fitness in implementing each component, could enable vendors to collectively self-assemble their respective existing solutions into a GCI consisting of a system of such agents. In a model in which each entity owns their own data, a GCI might coordinate massive decentralized queries to provide access to large data sets. By enabling users or intelligent useragents to spontaneously self-assemble into communities, a GCI could potentially enable collaboration with any dataset to achieve any outcome. Scaling collective reasoning through the assistance of intelligent agents in this way removes the current limit to the scale and speed at which a group can cooperate to find and evaluate solutions. Where current decision-systems might have the capacity to evaluate perhaps tens or hundreds of options, a GCI might evaluate trillions. Without the capacity to consider sufficient solutions (a sufficient region of solution space) to have the capacity to maximize outcomes, maximizing impact on collective outcomes (such as the sustainable development goals) cannot be reliably achievable.

In the individual human organism, the FMF models the functions of the body as transitions (paths) between points in the body's functional state space. The cognitive system represents all of these transitions in its conceptual space, so that the corresponding functions are available to the system of cognition (the mind). The cognitive system also defines semantic models to represent any objects in the conceptual space that the body might interact with. In a collective cognition (a GCI) the functions of the people in the collective are also modeled so those functions are available as internal group processes of the collective body. In addition, the functions of the products and services at their disposal are also modeled semantically so they can be made available as external processes to those internal group processes. For example, an internal process for an individual is dialing a number on a phone. The processes by which the phone connects the call are external to the individual. In the case of a collective, one internal process to the collective might involve one hundred thousand people selling access to a protected area in their mobile phones as a service. Some external process might negotiate purchase of this resource by some application developer so they could load test their application.

In order to be able to identify all the external services available to internal group processes, the GCI must develop a functional model of the potential interactions between group members (the collective body) and all external processes. In the case of the collective body the possible interactions are physical interactions through the five senses. In order to define all the functions that groups may achieve through such physical interactions, the collective intelligence must have the capacity to represent the physical functions of all individuals in the collective, as well as the capacity to represent the entities the collective interacts with. In order to be common among all individuals in the group this conceptual model must be human-centric rather than specific to any tool. Since this model must reside in the collective cognition it must be a conceptual model. A human-centric functional model defined in the conceptual space is a semantic model. Therefore, semantic modeling is a boundary condition for being able to cooperate within a GCI. In the same way that an individual's mind defines the available functional states (functional state space) of the body so that it can guide the body's interactions with the semantic model of the world it has constructed, a GCI must develop a functional model of the collective body to enable it to guide every individual in the collective through interactions with a 
semantic model of each user interface, as well as with each individual's personal Internet of Things (IoT), or other tools with which they might interact with their environment.

\section{Further Thoughts}

It's intuitively obvious that there is no tool that fits all jobs. But it is less intuitively obvious whether any individual decision-maker, or any system of decision-making (outside of one with general problem solving ability), can remove the systematic barriers discussed in this paper that are proposed to prevent groups from optimizing collective outcomes in the absence of General Collective Intelligence (GCI). These barriers have also been proposed in this paper to restrict any group to having an intelligence factor that is potentially more aligned with the interests of the individual intelligence of some subset of its members, rather than having a collective intelligence that is truly an emergent property of the group.

There may be a reason that no human decision-maker in the history of the earth, and no decisionmaking system has yet been able to solve poverty, environmental degradation, and other collective challenges facing us, as evidenced by the fact that such challenges still exist. In many cases [28], identifying these barriers requires a model of GCI. These barriers may not have even been conceived of by decision-makers or current decision-making systems (are invisible to them), and therefore fall outside of the choices any such individuals or systems are capable of making.

Rather than merely being a theoretical construct, this model of GCI is patterned after the balance in multicellular organisms between centralized prioritization of specific functions, and decentralized cooperation between cells to achieve those functions. This allows cells in organisms to tend towards a natural balance of collective well-being, so that the cooperation between them is stable and therefore sustainable [65],[66],[67]. In that multicellular organisms still exist over a billion years after their evolution, this cooperation has demonstrated stability and robustness against non-cooperators, such as cancer, bacteria, viruses and other entities that might attempt to break cooperation. Because while one organism may die, multicellular cooperation has remained over it's billion plus year history on earth.

Processes such as homeostasis, reproduction, evolution, or even reasoning, can be seen as processes by which humans can adapt to become more fit at any given function. Any adaptation that increases fitness is a decision that improves outcomes. In this way, humans as collections of multiple cells are decisionsystems designed to optimize collective outcomes for our cells. In that our various processes of adaptation for increased fitness have resulted in us becoming multi-celled organisms, this multicellular cooperation must be stable in that it conveys some fitness advantage that reliably incentivizes cells to form and maintain this cooperation.

The idea of a decision-making system that relies on such pervasive cooperation between humans as exists between human cells at first glance appears quite Utopian. Some individuals clearly do not cooperate. But if the cooperation between cells in all multicellular organisms can be distilled into a common pattern, it is the best solution nature has been able to develop in a billion plus years of trying all options it has encountered. And if that pattern also represents the inter-cellular cooperation that has produced reasoning, it is the only model of cooperating to achieve general problem solving ability capable of accumulating exponentially more value than it costs, that nature has created so far that we know of. From this perspective, finding a better model of collective decision-making than one which leverages this tried and tested pattern of nature may be highly improbable. Even the attempt to do so may be highly impractical.

Detailed requirements for some components of the Functional Modeling Framework (FMF) in which this model of GCI is represented have not yet been defined, so implementation of this full model GCI 
as defined within the FMF is not yet possible. However, enough important elements of this model (such as the principles of cooperation) can be implemented for such a model to provide the ability to gain significant increases in collective problem solving ability, as demonstrated through case studies referred to in this paper. This increased problem solving ability is demonstrated in those case studies in the potential for significant increases in collective impact. Given this potential increase in collective impact, and given that collective impact is involved in any problem faced by a group of people, GCI has critically important implications in virtually all industry sectors, since problems such as maximizing sustainability and other collective impacts, are collective ones.

The current iteration of this functional modeling approach and this GCI model are not intended to solve every problem, but instead to enable every problem definition to be combined into a single collective problem definition that becomes increasingly more fit at describing the world. And to enable every CI algorithm to be combined into a solution set that the collective becomes increasingly fit in using to navigate that problem space. Rather than seeking to replace existing CI solutions and other decisionmaking systems, this approach seeks to enable functional models of such solutions to be defined, so those solutions might be added to a such a library of collective reasoning processes.

\section{Conclusions}

Traditional models of CI ignore a number of constraints to optimal problem definition and optimal problem solving. One is the need for decentralization to balance the forces of centralization that constrain solutions so they solve the wrong problems. Using a functional modeling approach for representing the functions of the human system this paper has presented a model of collective cognition defined purely in terms of the dynamics between the cognitive functions. This common human-centric framework for understanding collective intelligence solutions and other decision-systems as elements of general collective intelligence is the first step in facilitating the large scale research collaboration that might be required to implement such models. The importance of these models to some existential collective problems creates the potential for collective investment in that implementation.

\section{References}

[1] Paul C. Nutt, Types of Organizational Decision Processes, Administrative Science Quarterly Vol. 29, No. 3 (Sep., 1984), pp. 414-450, DOI: 10.2307/2393033

[2] Oommen A (2014) Factors Influencing Intelligence Quotient. J Neurol Stroke 1(4): 00023. DOI: 10.15406/jnsk.2014.01.00023

[3] Andy E. Williams, A Functional Modeling Framework for Representing and Comparing Models of Consciousness or Cognition, under review

[4] J. Salminen, Collective intelligence in humans: A literature review, CoRR abs/1204.3401 (2012).

[5] Shweta Suran, Vishwajeet Pattanaik, and Dirk Draheim. 2020. Frameworks for Collective Intelligence: A Systematic Literature Review. ACM Comput. Surv. 53, 1, Article 14 (February 2020), 36 pages. DOI:https://doi.org/10.1145/3368986

[6] Solberg Søilen, Klaus, Making sense of the collective intelligence field: A review, 2019 (English), Journal of Intelligence Studies in Business, ISSN 2001-015X, E-ISSN 2001-015X, Vol. 9, no 2, p. 6-18 [7] Yu, C., Chai, Y. and Liu, Y. (2018), "Literature review on collective intelligence: a crowd science perspective", International Journal of Crowd Science, Vol. 2 No. 1, pp. 64-73.

[8] Javier Del Ser, Eneko Osaba, Daniel Molina, Xin-She Yang, Sancho Salcedo-Sanz, David Camacho, Swagatam Das, Ponnuthurai N. Suganthan, Carlos A. Coello Coello, Francisco Herrera, Bioinspired computation: Where we stand and what's next, Swarm and Evolutionary Computation, Volume 48, 2019, Pages 220-250, ISSN 2210-6502, https://doi.org/10.1016/j.swevo.2019.04.008. 
[9] A. W. Woolley, C. F. Chabris, A. Pentland, N. Hashmi, T. W. Malone, Evidence for a collective intelligence factor in the performance of human groups. Science 330, 686-688 (2010).

[10] Kahneman, D. (2012).Thinking, fast and slow.London: Penguin Books. Isbn: 0141033576.

[11] Andy E. Williams, The Case for General Collective Intelligence Rather than Artificial General Intelligence being the Most Important Human Innovation in the History and Future of Mankind, working paper.

[12] Overgaard M. (2017). The Status and Future of Consciousness Research. Frontiers in psychology, 8, 1719. https://doi.org/10.3389/fpsyg.2017.01719

[13] Caney, S., \& Hepburn, C. (2011). Carbon Trading: Unethical, Unjust and Ineffective? Royal Institute of Philosophy Supplement, 69, 201-234. doi:10.1017/S1358246111000282

[14] Mary J. Bortscheller, Equitable but Ineffective: How the Principle of Common but Differentiated

Responsibilities Hobbles the Global Fight against Climate Change, Sustainable Dev. L. \& Pol'y 49 (2009-2010)

[15] Hristos Doucouliagos, Martin Paldam, The ineffectiveness of development aid on growth: An update, European Journal of Political Economy, Volume 27, Issue 2, 2011, Pages 399-404, ISSN 01762680, https://doi.org/10.1016/j.ejpoleco.2010.11.004.

[16] Maranto, R.; Wai, J. Why Intelligence Is Missing from American Education Policy and Practice, and What Can Be Done About It. J. Intell. 2020, 8, 2.

[17] Brush, E. Inconvenient truths: pluralism, pragmatism, and the need for civil disagreement. J Environ Stud Sci (2020). https://doi.org/10.1007/s13412-020-00589-7

[18] The Council of Economic Advisers, Government Employment and Training Programs: Assessing the Evidence on their Performance, Published June 2019. Accessed on March 27, 2020 at https://www.whitehouse.gov/wp-content/uploads/2019/06/Government-Employment-and-TrainingPrograms.pdf

[19] Badman, R., Hills, T. T., \& Akaishi, R. (2020, March 4). Navigating Uncertain Environments: Multiscale Computation in Biological and Artificial Intelligence. https://doi.org/10.31234/osf.io/ced3t [20] Dagmar Monett, Colin W. P. Lewis, Kristinn R. Thórisson, On Defining Artificial Intelligence, Journal of Artificial General Intelligence, Volume 11: Issue 2, Introduction to the JAGI Special Issue, DOI: https://doi.org/10.2478/jagi-2020-0003 | Published online: 03 Mar 2020

[21] Lorenzo De Sio \& Till Weber (2020) Issue yield, campaign communication, and electoral performance: a six-country comparative analysis, West European Politics, 43:3, 720-745, DOI: 10.1080/01402382.2019.1655968

[22] Katrien Fransen, Niels Mertens, Stewart T. Cotterill, Gert Vande Broek \& Filip Boen (2020) From Autocracy to Empowerment: Teams with Shared Leadership Perceive their Coaches to be Better Leaders, Journal of Applied Sport Psychology, 32:1, 5-27, DOI: 10.1080/10413200.2019.1617370

[23] Lorenz, E.N.: Deterministic nonperiodic flow. J. Atmos. Sci. 20, 130 (1963)

[24] B. CHANDRASEKARAN, Representing function: Relating functional representation and functional modeling research streams, Artificial Intelligence for Engineering Design, Analysis and Manufacturing 2005, 19, 65-74, DOI: 10.10170S0890060405050079

[25] Zhang, W.Y., Tor, S.Y. \& Britton, G.A. , Managing modularity in product family design with functional modeling, The International Journal of Advanced Manufacturing Technology(2006) 30: 579. https://doi.org/10.1007/s00170-005-0112-z

[26] Eisenbart, B., Gericke, K., \& Blessing, L. (2013). An analysis of functional modeling approaches across disciplines. Artificial Intelligence for Engineering Design, Analysis and Manufacturing, 27(3), 281-289. doi:10.1017/S0890060413000280

[27] Zhengrong Cheng, Yongsheng Ma, A functional feature modeling method, Advanced Engineering Informatics, Volume 33, 2017, Pages 1-15, ISSN 1474-0346, https://doi.org/10.1016/j.aei.2017.04.003. [28] Tensa, M., Edmonds, K., Ferrero, V., Mikes, A., Soria Zurita, N., Stone, R., \& DuPont, B. (2019). Toward Automated Functional Modeling: An Association Rules Approach for Mining the Relationship 
between Product Components and Function. Proceedings of the Design Society: International Conference on Engineering Design, 1(1), 1713-1722. doi:10.1017/dsi.2019.177

[29] Sten Bay Jørgensen, Morten Lind, and Niels Jensen, Functional Modeling View on Product and Process Engineering in Design and Operations

Industrial \& Engineering Chemistry Research 201958 (26), 11129-11148

DOI: $10.1021 /$ acs.iecr.8b06338

[30] Zhao, Meng, et al. "A State-Behavior-Function Model for Functional Modeling of Multi-State Systems." Proceedings of the Institution of Mechanical Engineers, Part C: Journal of Mechanical Engineering Science, vol. 233, no. 7, Apr. 2019, pp. 2302-2317, doi:10.1177/0954406218791640. [31] von Neumann J, Morgenstern O (1944) Theory of Games and Economic Behavior. Princeton, NJ: Princeton University Press.

[32] Simon H (1955) A behavioral model of rational choice. Quarterly Journal of Economics 69: 99118.

[33] Philippe, T.W., \& Koehler, J.W. (2005). A Factor Analytical Study of Perceived Organizational Hypocrisy.

[34] Blanco-Fernández Y., Pazos-Arias J.J., Gil-Solla A., Ramos-Cabrer M., López-Nores M. (2008) Semantic Reasoning: A Path to New Possibilities of Personalization. In: Bechhofer S., Hauswirth M., Hoffmann J., Koubarakis M. (eds) The Semantic Web: Research and Applications. ESWC 2008.

Lecture Notes in Computer Science, vol 5021. Springer, Berlin, Heidelberg

[35] Franke, M., Jäger, G. Bidirectional Optimization from Reasoning and Learning in Games. J of Log Lang and Inf 21, 117-139 (2012). https://doi.org/10.1007/s10849-011-9151-z

Klinov P., Parsia B. (2008) Optimization and Evaluation of Reasoning in Probabilistic Description Logic: Towards a Systematic Approach. In: Sheth A. et al. (eds) The Semantic Web - ISWC 2008. ISWC 2008. Lecture Notes in Computer Science, vol 5318. Springer, Berlin, Heidelberg [36] Parra-Agudelo, L., Hee-jeong Choi, J., Foth, M. et al. Creativity and design to articulate difference in the conflicted city: collective intelligence in Bogota's grassroots organisations. AI \& Soc 33, 147158 (2018). https://doi.org/10.1007/s00146-017-0716-5

[37] M. Klein, "Achieving Collective Intelligence via Large-Scale On-line Argumentation," Second International Conference on Internet and Web Applications and Services (ICIW'07), Morne, 2007, pp. 58-58. doi: 10.1109/ICIW.2007.13, URL: http://ieeexplore.ieee.org/stamp/stamp.jsp?

$\mathrm{tp}=$ \&arnumber $=4222960$ \&isnumber $=4222896$

[38] Szuba T.., Polański P., Schab P., Wielicki P. (2011) On Efficiency of Collective Intelligence Phenomena. In: Nguyen N.T. (eds) Transactions on Computational Collective Intelligence III. Lecture Notes in Computer Science, vol 6560. Springer, Berlin, Heidelberg [39] S. Cho and R. G. Melhem, "On the Interplay of Parallelization, Program Performance, and Energy Consumption," in IEEE Transactions on Parallel and Distributed Systems, vol. 21, no. 3, pp. 342-353, March 2010.

[40] K. Kubota, A. Nakase, H. Sakai and S. Oyanagi, "Parallelization of decision tree algorithm and its performance evaluation," Proceedings Fourth International Conference/Exhibition on High Performance Computing in the Asia-Pacific Region, Beijing, China, 2000, pp. 574-579 vol.2. [41] Flores-Mendoza C., Ardila R., Rosas R., Lucio M.E., Gallegos M., Reátegui Colareta N. (2018) Intelligence, Problem Solving, and Creativity. In: Intelligence Measurement and School Performance in Latin America. Springer, Cham

[42] Funke, J.; Fischer, A.; Holt, D.V. When Less Is Less: Solving Multiple Simple Problems Is Not Complex Problem Solving-A comment on Greiff et al. (2015). J. Intell. 2017, 5, 5.

[43] Fluid intelligence and cognitive segmentation

John Duncan, Daphne Chylinski, Daniel J. Mitchell, Apoorva Bhandari

Proceedings of the National Academy of Sciences May 2017, 114 (20) 5295-5299; DOI:

10.1073/pnas.1621147114 
[44] Paetow K., Schmitt M., Malsch T. (2005) Scalability, Scaling Processes, and the Management of Complexity. A System Theoretical Approach. In: Fischer K., Florian M., Malsch T. (eds) Socionics. Lecture Notes in Computer Science, vol 3413. Springer, Berlin, Heidelberg [45] Cabot J., Clarisó R., Brambilla M., Gérard S. (2018) Cognifying Model-Driven Software Engineering. In: Seidl M., Zschaler S. (eds) Software Technologies: Applications and Foundations. STAF 2017. Lecture Notes in Computer Science, vol 10748. Springer, Cham [46] Kounev S. et al. (2017) The Notion of Self-aware Computing. In: Kounev S., Kephart J., Milenkoski A., Zhu X. (eds) Self-Aware Computing Systems. Springer, Cham [47] Zorrilla M., García-Saiz D. (2015) Meta-Learning Based Framework for Helping Non-expert Miners to Choice a Suitable Classification Algorithm: An Application for the Educational Field. In: Núñez M., Nguyen N., Camacho D., Trawiński B. (eds) Computational Collective Intelligence. Lecture Notes in Computer Science, vol 9330. Springer, Cham [48] García-Saiz, Diego and Zorrilla, Marta. 'A Meta-learning Based Framework for Building Algorithm Recommenders: An Application for Educational Arena'. 1 Jan. 2017 : 1449 - 1459. [49] Bassey Ekpe, The United Nations and the Rationale for Collective Intelligence, Cambria Press, 2009, ISBN 1604976144, 9781604976144

[50] Computational Collective Intelligence -- Technologies and Applications: 6th International Conference, ICCCI 2014, Seoul, Korea, September 24-26, 2014, Proceedings

Volume 8733 of Lecture Notes in Computer Science, Lecture Notes in Artificial Intelligence Editors Dosam Hwang, Jason J. Jung, Ngoc Thanh Nguyen, Springer, 2014, ISBN 3319112899 , 9783319112893

[51] Advances in Collective Intelligence 2011, Volume 113 of Advances in Intelligent and Soft Computing, Editors Jörn Altmann, Ulrike Baumöl, Bernd Krämer, Springer Science \& Business Media, 2012, ISBN 3642253210, 9783642253218

[52] Javier Del Ser, Eneko Osaba, Daniel Molina, Xin-She Yang, Sancho Salcedo-Sanz, David Camacho, Swagatam Das, Ponnuthurai N. Suganthan, Carlos A. Coello Coello, Francisco Herrera, Bioinspired computation: Where we stand and what's next, Swarm and Evolutionary Computation, Volume 48, 2019, Pages 220-250, ISSN 2210-6502, https://doi.org/10.1016/j.swevo.2019.04.008.

[53] Shweta Suran, Vishwajeet Pattanaik, and Dirk Draheim. 2020. Frameworks for Collective Intelligence: A Systematic Literature Review. ACM Comput. Surv. 53, 1, Article 14 (February 2020), 36 pages. DOI:https://doi.org/10.1145/3368986

[54] Solberg Søilen, Klaus, Making sense of the collective intelligence field: A review, 2019 (English), Journal of Intelligence Studies in Business, ISSN 2001-015X, E-ISSN 2001-015X, Vol. 9, no 2, p. 6-18 [55] Yu, C., Chai, Y. and Liu, Y. (2018), "Literature review on collective intelligence: a crowd science perspective", International Journal of Crowd Science, Vol. 2 No. 1, pp. 64-73.

https://doi.org/10.1108/IJCS-08-2017-0013

[56] Martie G. Haselton, Gregory A. Bryant, Andreas Wilke, David A. Frederick, Andrew Galperin, Willem E. Frankenhuis, and Tyler Moore (2009). Adaptive Rationality: An Evolutionary Perspective on Cognitive Bias. Social Cognition: Vol. 27, Special Issue: Rationality Restored, pp. 733-763. https://doi.org/10.1521/soco.2009.27.5.733

[57] Haselton, M.G., Nettle, D. and Murray, D.R. (2015). The Evolution of Cognitive Bias. In The Handbook of Evolutionary Psychology, D.M. Buss (Ed.). doi:10.1002/9781119125563.evpsych241 [58] Nguyen VT, Young B, Ravaud P, Naidoo N, Benchoufi M, Boutron I. Overcoming Barriers to Mobilizing Collective Intelligence in Research: Qualitative Study of Researchers With Experience of Collective Intelligence. J Med Internet Res. 2019;21(7):e13792. Published 2019 Jul 2.

doi:10.2196/13792

[59] Andy E. Williams, The Need for General Collective Intelligence in Addressing the Sustainable Development Goals, under review (2020) 
[60] A. E. Williams, Defining Functional Models of Collective Intelligence Solutions to Create a Library that a General Collective Intelligence can Use to Increase General Problem Solving Ability, 2019. Under review.

[61] A. E. Williams, The Design of the Social Impact Marketplace as a Partial Implementation of a General Collective Intelligence (GCI) Platform, working paper

[62] Christoffer Levandowski, Jakob R Müller, Ola Isaksson, Modularization in Concept Development Using Functional Modeling, Transdisciplinary Engineering: Crossing Boundaries: Proceedings of the 23rd ISPE Inc. International Conference on Transdisciplinary Engineering October 3 - 7, 2016 [63] P. M. Krafft, A simple computational theory of general collective intelligence, Topics in Cognitive Science 11 (2019) 374-392.25

[64] Brennan, T. J., \& Lo, A. W. (2012). An evolutionary model of bounded rationality and intelligence. PloS one, 7(11), e50310. https://doi.org/10.1371/journal.pone.0050310

[65] Driscoll, W., Travisano, M. Synergistic cooperation promotes multicellular performance and unicellular free-rider persistence. Nat Commun 8, 15707 (2017). https://doi.org/10.1038/ncomms15707 [66] Jay M. Biernaskie and Stuart A. West 2015Cooperation, clumping and the evolution of multicellularityProc. R. Soc. B.28220151075, http://doi.org/10.1098/rspb.2015.1075

[67] Michod, R., Roze, D. Cooperation and conflict in the evolution of multicellularity. Heredity 86, 17 (2001). https://doi.org/10.1046/j.1365-2540.2001.00808.x

[68] Chikersal, Prerna, et al. "Deep Structures of Collaboration: Physiological Correlates of Collective Intelligence and Group Satisfaction." Proceedings of the 20th ACM Conference on ComputerSupported Cooperative Work and Social Computing (CSCW 2017). 2017.

[69] Macphail, E. (1987). The comparative psychology of intelligence. Behavioral and Brain Sciences, 10(4), 645-656. doi:10.1017/S0140525X00054984

[70] Batson, C. D., Ahmad, N., Yin, J., Bedell, S. J., Johnson, J. W., \& Templin, C. M. (1999). Two

Threats to the Common Good: Self-Interested Egoism and Empathy-Induced Altruism. Personality and Social Psychology Bulletin, 25(1), 3-16. https://doi.org/10.1177/0146167299025001001

[71] Simon, H. (1957). "A behavioral model of rational choice," in Models of Man, Social and Rational: Mathematical Essays on Rational Human Behavior in a Social Setting. New York: Wiley. [72] Lebiere Christian, Anderson John, Cognitive Constraints on Decision Making under Uncertainty, Frontiers in Psychology, Vol 2, 2011, page 305, DOI: 10.3389/fpsyg.2011.00305, ISSN=1664-1078 [73] Knapp, M. S., Barnard, K., Brandon, R. N., Gehrke, N. J., Smith, A. J., \& Teather, E. C. (1993). University-based preparation for collaborative interprofessional practice. In L. Adler \& S. Gardner (Eds.), The politics of linking schools and social services. Washington, DC: The Falmer Press.

[75] Das, T. and Teng, B.-S. (1999), Cognitive Biases and Strategic Decision Processes: An Integrative Perspective. Journal of Management Studies, 36: 757-778. doi:10.1111/1467-6486.00157 